\title{
Remote sensing of life: polarimetric signatures of photosynthetic pigments as sensitive biomarkers
}

\author{
Svetlana V. Berdyugina ',2, Jeff R. Kuhn ${ }^{3}$, David M. Harrington ${ }^{1,3}$, Tina Šantl-Temkiv ${ }^{4}$ \\ and $\mathrm{E}$. John Messersmith ${ }^{3}$ \\ ${ }^{1}$ Kiepenheuer Institut für Sonnenphysik, Schoeneckstr. 6, Freiburg 79104, Germany \\ email: sveta@kis.uni-freiburg.de \\ ${ }^{2}$ NASA Astrobiology Institute, University of Hawaii, Institute for Astronomy, 2680 Woodlawn Dr, Honolulu, HI 96822, \\ USA \\ ${ }^{3}$ Institute for Astronomy, University of Hawaii, 34 Ohia Ku St, Pukalani, Maui, HI 96768, USA \\ ${ }^{4}$ Stellar Astrophysics Center, Department of Physics and Astronomy, Aarhus University, Aarhus, Denmark
}

\begin{abstract}
We develop a polarimetry-based remote-sensing method for detecting and identifying life forms in distant worlds and distinguishing them from non-biological species. To achieve this we have designed and built a bio-polarimetric laboratory experiment $\mathrm{BioPol}$ for measuring optical polarized spectra of various biological and non-biological samples. Here we focus on biological pigments, which are common in plants and bacteria that employ them either for photosynthesis or for protection against reactive oxygen species. Photosynthesis, which provides organisms with the ability to use light as a source of energy, emerged early in the evolution of life on Earth. The ability to harvest such a significant energy resource could likely also develop on habited exoplanets. Thus, we investigate the detectability of biomolecules that can capture photons of particular wavelengths and contribute to storing their energy in chemical bonds. We have carried out laboratory spectropolarimetric measurements of a representative sample of plants containing various amounts of pigments such as chlorophyll, carotenoids and others. We have also measured a variety of nonbiological samples (sands, rocks). Using our lab measurements, we have modelled intensity and polarized spectra of Earth-like planets having different surface coverage by photosynthetic organisms, deserted land and ocean, as well as clouds. Our results demonstrate that linearly polarized spectra provide very sensitive and rather unambiguous detection of photosynthetic pigments of various kinds. Our work paves the path towards analogous measurements of microorganisms and remote sensing of microbial ecology on the Earth and of extraterrestrial life on other planets and moons.
\end{abstract}

Received 10 May 2014, accepted 19 February 2015, first published online 20 May 2015

Key words: biopigments, biosignatures, Earth-like planets, exoplanets, photosynthesis, remote sensing of life, spectropolarimetry

\section{Introduction}

The production of organic compounds by reducing carbon from carbon dioxide directly or indirectly supports all life on Earth. First terrestrial organisms $(\sim 3.8 \mathrm{Ga})$ used chemical sources of energy in order to produce organic compounds. The origin of oxygenic, water-based photosynthesis on early Earth (from $\sim 3.6 \mathrm{Ga}$ ) gave organisms access to a much more abundant energy source, allowing the biosphere to achieve orders of magnitude higher organic productivity (Des Marais 2000), which came to support life as we know it today. This evolutionary innovation was also important in terms of liberating the primary production from its dependence on abiotic chemical compounds, e.g. in hydrothermal vents, and made the life globally distributed and dependent only on sunlight, water and nutrients (Des Marais 2000). It had led to dramatically increased biomass, the global distribution of biosphere, and at the same time resulted in abundant molecular biosignatures. Both atmospheric oxygen buildup (Des Marais et al. 2002) and flux spectral signatures of biological pigments (Kiang et al. 2007a, b) arising from photosynthesis have been proposed as biosignatures on extra-solar planets. Due to the accessibility and amount of energy provided by the stellar radiation, it seems natural for life to evolve the photosynthetic ability of utilizing it as an energy source also on other planets.

Capturing stellar energy by photosynthetic organisms relies on complex assemblies of biological pigments which are organized in a reaction centre (RC), converting light energy into chemical energy, and an accessory light-harvesting complex (LHC), helping increase the efficiency of harvesting light (Scholes et al. 2011). While chlorophyll $a(\mathrm{Chl} a)$ is the primary pigment of the $\mathrm{RC}$ in cyanobacteria, algae and plants, there are up to 200 accessory and secondary (synthesized) biopigments in LHC, including various forms of chlorophyll (Chl $b, c$ and $d$ ), carotenoids (Car), anthocyanins (Ant), phycobiliproteins (PBP), etc. The spectral sensitivity of biopigments is such that they absorb almost all light in the visible range (Kiang 
et al. 2007a, and references therein). This enabled even the human eye retina which evolved by utilizing photosynthesis.

We focus here on a polarimetric study of photosynthetic pigments of higher plants, which evolved from cyanobacteria and green algae. The absorption peaks of chls occur in the red and the blue part of the visual spectrum (Chl $a, b, c$ and $d$ have absorption peaks at slightly different wavelengths) with an intermediate small reflection peak at green wavelengths, which defines the green colour of plants and cyanobacteria in the visible. Different carotenoids absorb blue and green light, which results in yellow, orange and reddish pigmentation of plant parts. Both biopigment types reflect the near infrared (IR) light: one can see with IR goggles that green plants are supremely bright in the near IR when illuminated by sunlight. During evolution pigment absorbance can shift substantially due to small changes in their chemical structure (e.g. Blankenship \& Hartman 1998), and the evolution of their absorbance features was likely constrained by their spectral environment (Kiang et al. 2007a). For example, the red peak absorbance of $\mathrm{Chl}$ in $\mathrm{RC}$ of higher plants is near the peak incident photon flux at Earth's surface. The RC electronic structure poses additional constrains on the absorption features of the pigments, so that photons used to excite electrons in the $\mathrm{Chl}$ of the RC should carry sufficient energy to enable the synthesis of organic compounds, i.e. storage of chemical energy.

Kiang et al. (2007b) proposed that photosynthetic pigments on planets around stars of different effective temperatures (spectral classes F, G, K and M) might be adapted to the spectral energy distribution of the host star as well as to the atmospheric transmission of the planet. They calculated the incident spectral photon fluxes on surfaces of different exoplanets and identified bands of photosynthetically relevant radiation as a major driver of pigment evolution. While on Earth orbiting a $\mathrm{G} \operatorname{star}\left(T_{\mathrm{eff}}=5800 \mathrm{~K}\right)$, colours of land plants are defined mainly by pigments absorbing predominantly in the visible blue and red (i.e. green and yellow plants dominate), on planets with Earth-like atmospheres orbiting $\mathrm{F}\left(T_{\text {eff }}=7000 \mathrm{~K}\right)$ or $\mathrm{M}\left(T_{\text {eff }}=3500 \mathrm{~K}\right)$ stars, photosynthetic pigments may have peak absorbance in the blue and near IR part of the spectrum, respectively (Kiang et al. 2007b). It is worth also to note that a lack of blue photons in cool $\mathrm{M}$ stars will probably require a more complex mechanism for splitting water molecules into hydrogen and oxygen involving three or four photons instead of two as it occurs in terrestrial organisms. Therefore, understanding properties of radiation reflected from various photosynthetic organisms may help to identify such life forms on distant planets. This is the primary goal of our study.

Reflectance flux and polarization spectra of numerous photosynthetic organisms at various angles were measured in laboratories, in situ, and from space but understanding radiation transport within these organisms is still incomplete. Studies of optical properties of various leaves and inorganic samples have been conducted for several decades (e.g. Pospergelis, 1969, Grant et al. 1993; Brakke 1994; Jacquemoud \& Ustin 2001; Raven et al. 2002; Suomalainen et al. 2009; Peltoniemi et al. 2009). The POLDER satellite provided narrowband measurements of various landscape features which were used for agricultural or ecological studies (e.g. Breon et al. 1995; Sridhar et al. 2005). However, the public POLDER database provides only near IR band linear polarization measurements which can be used to characterize only specular reflection from various vegetation where its reflected flux (but not polarization) is the largest. It was also proposed that chl could serve as a potential biosignature on exoplanets (e.g. Wolstencroft \& Breon 2005; Kiang et al. 2007a; Stam 2008). Sterzik et al. (2012) have investigated Earth shine reflected from a dark Moon surface as a proxy to exoplanets and looked for a trace of the $\mathrm{Chl}$ signature in linearly polarized spectra. Early studies of Pospergelis (1969) followed by Wolstencroft (1974) and others presented measurements of full Stokes and circular polarization of leaves and mineral samples. Sparks et al. (2009) continued this investigation with microbes. It was found that a unique circular polarization signature associated with homohirality in living organisms is extremely weak $\left(5 \times 10^{-4}\right.$ at most under laboratory conditions) which would require unrealistic exposure time with a gigantic space telescope to be detected on distant planets, but it can perhaps be searched for in the Solar system objects.

The goal of this paper is to extend experimental and theoretical spectropolarimetric studies of biopigments in order to evaluate their potential for remote sensing of life on other planets. In contrast to other studies, we focus on strong linear polarization signatures (several tens per cent, see Section 'Biological samples') associated with absorption by various biopigments in the visible rather than on weak specular polarization in the near IR (a few percent, e.g. POLDER data) or circular polarization $(<0.05 \%$, Sparks et al. 2009). We show that a corresponding polarized biosignature from distant planets should be searched for in spectral regions where we expect alien photosynthetic organisms to absorb the most, and not where they could reflect most of the incident flux.

Ability to detect weak signals from astronomical objects and distinguish them from overwhelmingly bright background radiation is often enhanced by employing polarimetry (if objects are polarized). This is because polarimetry is an intrinsically differential technique (see Section 'Laboratory setup') and, therefore, allows for significantly higher contrast as compared with flux measurements. For instance, if both the flux and spectral composition of the object and its background are hypothetically identical, they could never be distinguished with imaging, photometry or spectroscopy. However, if at the same time the object is polarized and the background is not (or it may be polarized in a different way), polarimetric measurements of the flux, spectra or direct images allow for removal of an unpolarized background and 'seeing' the object directly which otherwise would not be seen. This 'trick' works independently on the signal-to-noise ratio (SNR), but the latter determines the contrast level at which the object and its background can be distinguished. The most sensitive polarimetric measurements of an astronomical object (Sun) were made at a few parts in ten million level by Kemp et al. (1987) in broad spectral bands from an entire solar disk, but current instruments can achieve so far polarimetric sensitivity of a few parts in million for the Sun and Vega only (Stenflo et al. 2000; Hough et al. 2006). Recently, it was demonstrated that polarized reflected 
light can be detected from exoplanetary atmospheres even if the planet is not spatially resolved from the star (Berdyugina et al. 2008, 2011). First measurements of the planet geometrical albedo at several wavelengths using polarimetry with groundbased telescopes by Berdyugina et al. (2011) has revealed a blue colour of the planet, which was subsequently confirmed by observations with the Hubble space telescope (Evans et al. 2013). This opens a door for investigating colours and composition of exoplanetary atmospheres and surfaces with the polarimetric sensitivity of at least $10^{-5}$, i.e. ten parts in million (Piirola et al. 2014) using modest ground-based facilities for brighter targets or future large ground-based and space telescopes for fainter and smaller planets, and/or in relatively narrow spectral bands. So far, only Kepler mission achieved the $10^{-5}$ relative sensitivity in unpolarized light on some targets while integrating flux over the broad spectral range $400-900 \mathrm{~nm}$ during many hours or even days (e.g. Demory et al. 2011). Spatially resolved polarimetric imaging of planetary systems does not require high polarimetric accuracy and, in principle, is capable of detecting reflected light from exoplanets at larger distances from the central star, but this technique requires a larger telescope aperture to resolve the planet from the star and detect reflected photons. For instance, SPHERE at VLT (Beuzit et al. 2010) and GPI at Gemini (Perrin et al. 2015), both are $8 \mathrm{~m}$ aperture telescopes, will perhaps be able to detect a few brightest exoplanets in the Solar neighbourhood. Therefore, extremely large telescopes are needed to detect distant Earth-like planets (see Section 'Polarized spectra of habitable earth-like planets').

We have designed and built a BioPol instrument in order to carry out laboratory spectropolarimetric measurements of organisms carrying different photosynthetic and nonphotosynthetic pigments. First results of this experiment were reported by Berdyugina (2012). We have searched for and identified spectropolarimetric signatures of biological pigments, which can be employed for remote sensing of life forms on the Earth and in extraterrestrial environments. Then, we have incorporated the spectropolarimetric information obtained in our laboratory studies into models of Earth-like planets with various coverages of land, ocean, vegetation and clouds.

The results presented in this paper (1) significantly broaden the variety of measured samples for astronomical applications which so far did not include a variety of tropical plants, and (2) demonstrate the advantage of linear polarization associated with absorption by photosynthetic biopigments as compared with previous circular polarization measurements or near-IR specular reflection linear polarization. This allows us to justify a sensitive biomarker for distant detection of life, which can be distinguished from non-biological sources. We also foresee applications of the same technique for identification of microorganisms in the laboratory, various terrestrial environments and on the surface of another planet in the Solar system.

\section{Laboratory setup}

A schematic of our laboratory experiment BioPol is shown in Fig. 1. A sample piece was fixed on a glass slide, which was illuminated by an unpolarized light source (see below) at

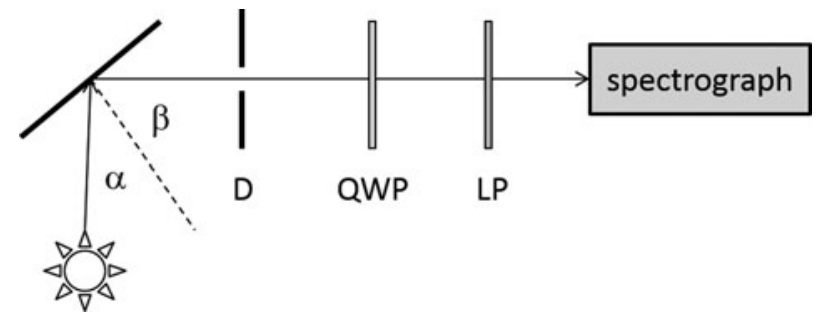

Fig. 1. A schematic of the laboratory setup used for measuring BRDF-PS from biological and non-biological samples at ATRC, IfA, Maui. The LP was used to measure linear polarization. An optional QWP was added to the setup to obtain circular polarization.

different light incident angles $\alpha$. Background light sources were eliminated during measurements. The reflectance angle $\beta$ is the angle between the normal to the sample plane and the direction to the detector. Both the sample and the light source were mounted on motorized rotational stages controlled by our software, so bidirectional reflectance distribution function (BRDF) of polarized spectra (PS) could be measured at all possible angles $\alpha$ and $\beta$ within the plane of Fig. 1. A diaphragm (D) selects a limited area of the sample, from which the reflected light is recorded. The reflected light was modulated by a quarter-wave-plate (QWP) or linear polarizer (LP) to obtain measurements of all polarization states expressed via four Stokes parameters: intensity $I$, linear polarization $Q$ and $U$, and circular polarization $V$. The Stokes parameters are defined through time averaged square amplitudes of the electric field vector in order to characterize its preferred space orientation with respect to the light propagation direction (see, e.g. Jackson 1998). In practice, Stokes parameters are measured as a difference between intensity spectra obtained at different angles of the LP and QWP. We have made measurements at four LP angles of $0^{\circ}$ and $90^{\circ}(I \pm Q)$ and $45^{\circ}$ and $135^{\circ}(I \pm U)$ in order to obtain normalized $Q / I$ and $U / I$ spectra. Also, $I \pm V$ spectra were measured with QWP modulation at the same four angles for redundancy to obtain normalized V/I. The modulated signal was transmitted through a fibre to a spectrograph (Ocean Optics, QE6500) with a detector that digitized and stored the spectra. The acquisition procedure was fully automated with an in-house developed software. The spectral resolution was $5 \mathrm{~nm}$, and the wavelength range was $350-1100 \mathrm{~nm}$, although at wavelengths shorter $400 \mathrm{~nm}$ SNR was low. Our QUV system is right handed in the direction toward the detector, and $Q$ is positive perpendicular to the optical table surface at which all the experiment elements are mounted.

The optical elements of this setup were first used for calibrating a new night-time polarimeter InnoPol and are described more thoroughly by Harrington et al. (2014). The input polarizer (for calibration) and analysing polarizer are both Moxtek ultra-broad band wire grid polarizers. These polarizers achieve contrast ratios above 1000 at $300 \mathrm{~nm}$ rising to 10000 at 2000 $\mathrm{nm}$ on a fused silica substrate with very flat transmission functions. Our rotation stages have $\leq 0.02^{\circ}$ repeatability with 400 steps per degree motion sensitivity in closed loop control. We have calibrated the setup for degree of polarization, transmission and input light source polarization in several steps. 
First, the analysing polarizer is referenced to the optical bench horizontal by finding the minimum transmitted intensity when crossed with the input polarizer in a square mount hard referenced directly on the optical bench. We find the minimum within a few steps positional error. Given mechanical mounting tolerances of standard optical breadboard components, our horizontal reference should be accurate to within $1^{\circ}$.

Second, the input polarizer is mounted on the rotation stage and a series of a few thousand spectra are recorded while the input polarizer and the analysing polarizer rotate through $360^{\circ}$ with respect to each other. A function including the input light source degree of polarization, contrast ratio and transmission functions is fit to these spectra. The input light source degree of polarization is $<1 \%$ across our wavelength range, and we achieve contrast ratios of at least a few hundred in our typical configuration between crossed polarizers.

The optical quarter wave plate retarder is then calibrated between crossed polarizers following a similar procedure. Several hundred spectra are recorded while the QWP is rotated through $360^{\circ}$, the analysing polarizer is rotated through $360^{\circ}$ and the input polarizer is rotated through $360^{\circ}$ for many independent combinations of input rotation angles. This allows us to fit a few simple functions of wavelength for the QWP fast axis orientation, retardance, input light source degree of polarization, optic transmission functions and lamp spectrum.

Our light source is fibre coupled with a $200 \mu \mathrm{m}$ core which is then collimated by a Thor Laboratories achromatic lens assembly. The input fibre source was a two-channel fibre combiner fed by two light sources. Both light sources were typical white light bulbs with different effective temperatures that were colour filtered to provide 'blue' and 'red' colour balance across a wide spectral range. Despite these efforts, the light source spectral distribution within the measured wavelength range was not truly 'white'. Additionally, the quantum efficiency of the Ocean Optics spectrograph peaks around 600 $\mathrm{nm}$ delivering lower SNR at wavelengths shorter than 400 $\mathrm{nm}$. To remove the light source spectral distribution, we made reflectance measurements of an ordinary office white paper. The reflectance spectra of our samples presented here are relative to this white paper, assuming that its albedo is 0.87 and wavelength independent. Therefore, our reflectance spectra are accurate within a few per cent only, which however does not affect our conclusions (see Section 'Polarized spectra of habitable earth-like planets'). We note however that this white paper calibration is irrelevant to polarization measurements which are intrinsically differential and are not affected by the light source spectral information (except for the SNR). To assure that the incident light is unpolarized, a spatial scrambler (depolarizer, wedged retarder) was mounted in front of the fibre collimating lens. This adds additional spatial scrambling of the potential input polarization which is subsequently spatially averaged over the illuminated region of the sample.

After calibration and data analysis we have extracted the Stokes parameter spectra. Measurements of the background light and darks were also made. The polarimetric sensitivity of our measurements is about $0.1 \%$. This sensitivity is more
Table 1. Photosynthetic organisms and inorganic samples measured in our experiment

\begin{tabular}{lll}
\hline$\#$ & Sample & Colour, texture \\
\hline Plant leaves & & \\
L1 & Eriobotrya japonica & Dark green, glossy \\
L2 & Eriobotrya japonica & Green/yellow, glossy \\
L3 & Tibouchina urvilleana & Green, hairy \\
L4 & Psidium cattleianum & Green, glossy \\
L5 & Evergreen & Green, matte \\
L6 & Iresine herbstii & Red/green, matte \\
L6d & Iresine herbstii & Red/green, dry \\
L7 & Cordyline fruticosa & Red/green, glossy \\
L8 & Bougainvillea spp. & Purple/green, matte \\
Flower petals & & \\
P1 & Tropaeolum & Yellow, matte \\
P2 & Tropaeolum & Orange, matte \\
P3 & Hibiscus rosa-sinensis & Red, glossy \\
P4 & Tibouchina urvilleana & Purple, matte \\
Inorganic samples & \\
S1 & Sand, New Mexico & White, gypsum \\
S2 & Sand, Western Australia & Yellow \\
S3 & Sand, Central Australia & Red \\
S4 & Sand, Central Sahara & Light brown \\
R1 & Rock 1, Hawaii & Grey/brown \\
R2 & Rock 2, Hawaii & Black lava \\
\hline
\end{tabular}

than enough for modelling linear polarization signals from exoplanets (see Section 'Polarized spectra of habitable earthlike planets'). The circular polarization signal due to homohirality $(0.05 \%)$ could not be detected with this sensitivity, so in this paper we only discuss results for linear polarization.

All samples were first measured to find an optimal $\alpha$ and $\beta$ angle combination resulting in a higher polarization degree. We found that reflection at $\alpha=\beta=50^{\circ}$ is a reasonable choice for our comparative analysis, which is actually close to the Brewster angle for the air-water interface. In this paper we present results measured at these angles. A complete data set of BRDF-PS will be presented in a separate paper. Thus, our results refer to an approximately maximum possible effect to be seen in exoplanets. We note however that the observed polarization degree also depends on the spectral resolution, and it can be higher in some cases when a spectral signature is well resolved.

\section{Biological samples}

We have measured more than a dozen of plant samples, including various green leaves, flower petals, fruit and vegetable pieces. These plants were collected and measured in Hawaii, and they represent a sample of tropical plants which complement to previous studies of mid-latitude forest and agricultural plants and grasses. In fact, our goal is to extract and understand a generalized polarized photosynthetic signature of terrestrial organisms, and we are not interested in studying particular species. Therefore, our selection is relatively random and was guided only by sampling a variety of biopigments in plants. These biopigments occur also in plants and other photosynthetic organisms outside Hawaii. Table 1 contains 

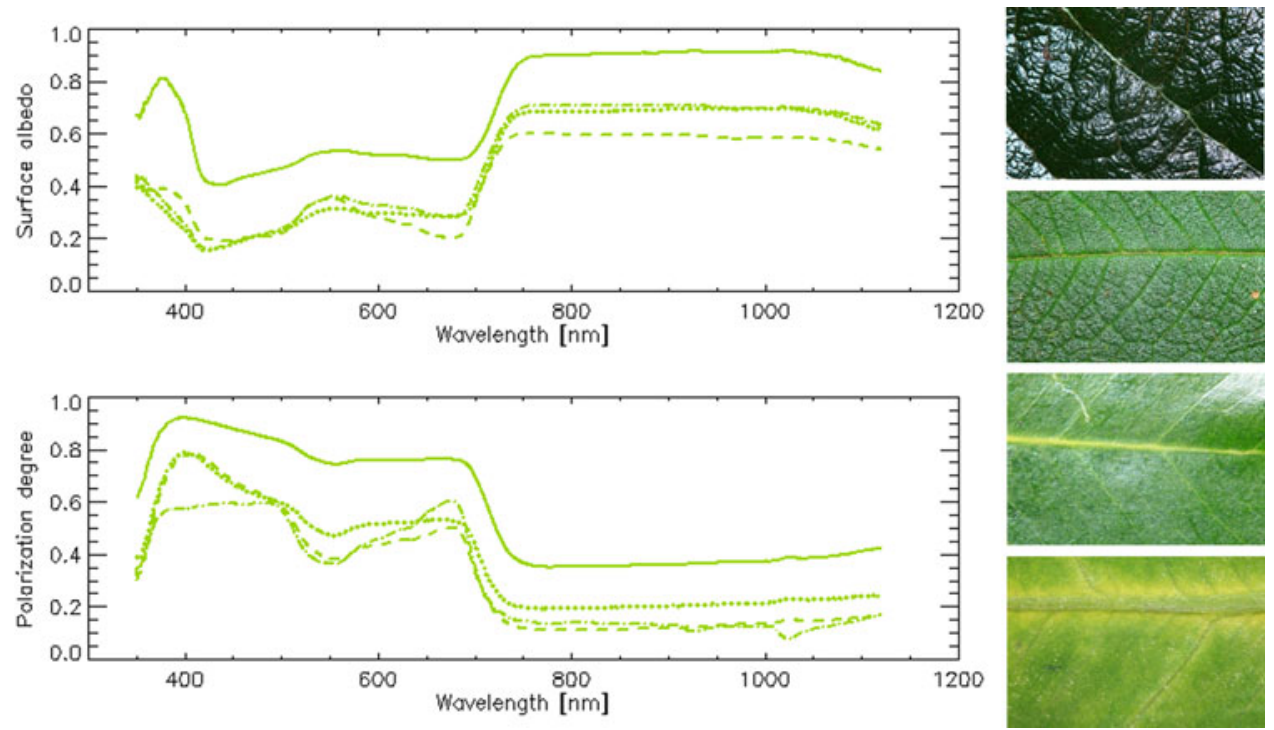

Fig. 2. Reflectance and polarized spectra of green leaves and images of the samples (top to bottom): L1-solid, L3-dotted, L4-dashed, L5dashed-dotted (see Table 1). Images of the plants are provided to illustrate their appearance. These are original measured plants, and the measured area is within these images. Note that the higher polarization occurs at the wavelengths where Chl most efficiently absorbs photons. The so-called 'red edge' near $700 \mathrm{~nm}$ is clearly visible. Also, polarization and reflectance are elevated if the surface of the leaf is glossy, but this additional intensity and polarization is practically wavelength independent (see white specular reflection on leaves on the right).
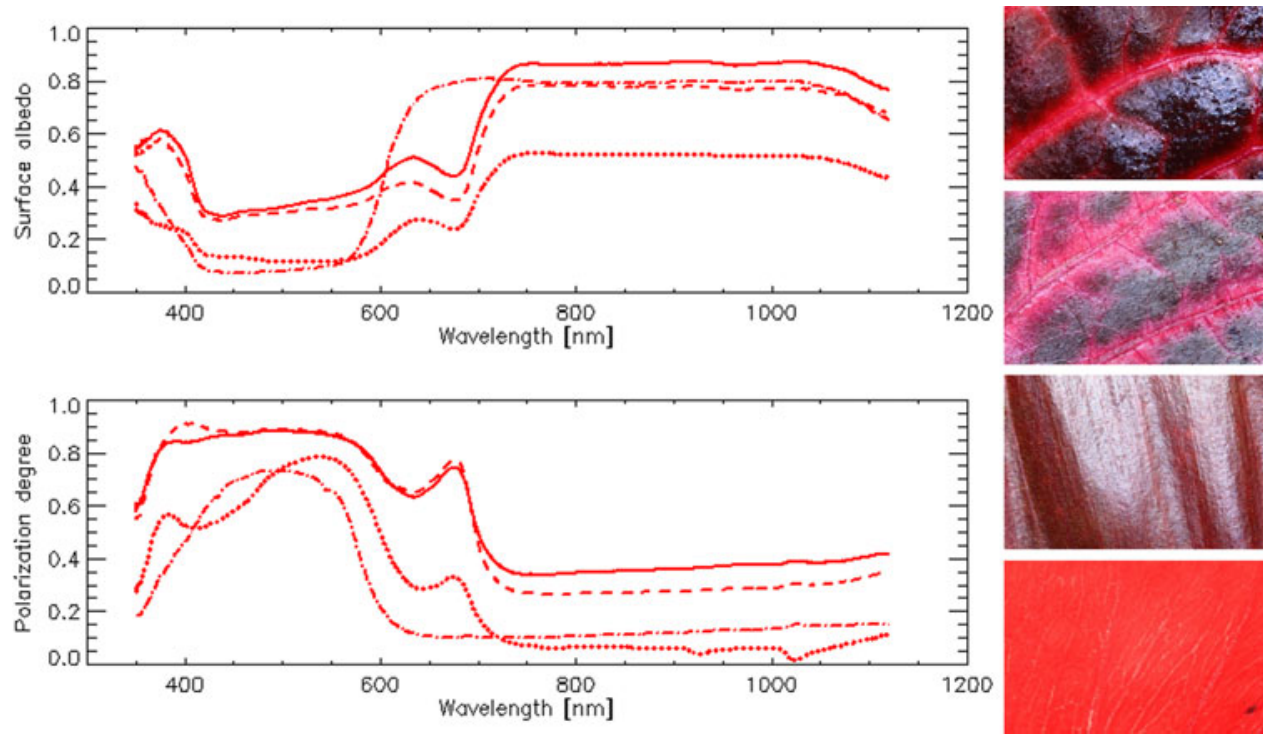

Fig. 3. Reflectance and polarized spectra of red leaves with green inclusions and a red petal (images of the samples top to bottom): L6-solid, L6ddotted, L7-dashed, P3-dashed-dotted (see Table 1). The presence of chl and other biopigments is revealed by both absorption and polarization. The specular reflection effect is the same as before.

information on the species analysed here and their properties. The leaf and petal samples were measured afresh, just after collecting them from parent plants. The measured signal was reproducible within a few hours after sample collection. Evergreen, sturdy leaves maintained their properties for days, except some loss of humidity which did not affect our optical measurements where no strong water band absorption could be seen. Some leaves were collected already in a decaying state (e.g. L2 and L6d) in order to measure their pigment composition with respect to the fresh leaf (L1 and L6). The reflected light was always measured from the upper (adaxial) leaf side, i.e. the one normally facing the Sun.

The reflectance and polarization degree spectra for our biological samples are shown in Figs. 2-5. As mentioned in Section 'Laboratory setup', these are spectra with the largest polarization recorded in our experiment. Remarkably, different parts of plants with the same pigment showed very similar spectral dependence of the reflectance and polarization 

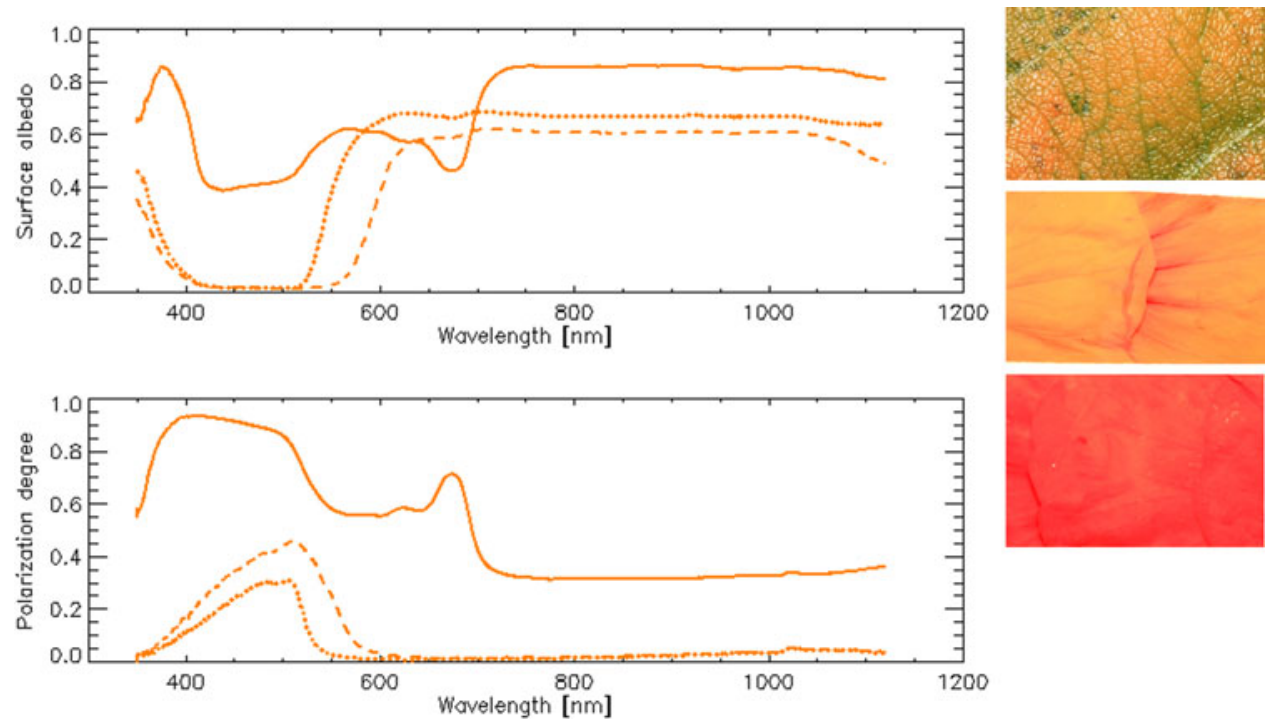

Fig. 4. Reflectance and polarized spectra of a yellow leaf with green inclusions and of yellow and orange flower petals (images of the samples top to bottom): L2-solid, P1-dotted, P2-dashed (see Table 1). The presence of chl and carotenoids is revealed by both absorption and polarization. The specular reflection effect is the same as before.
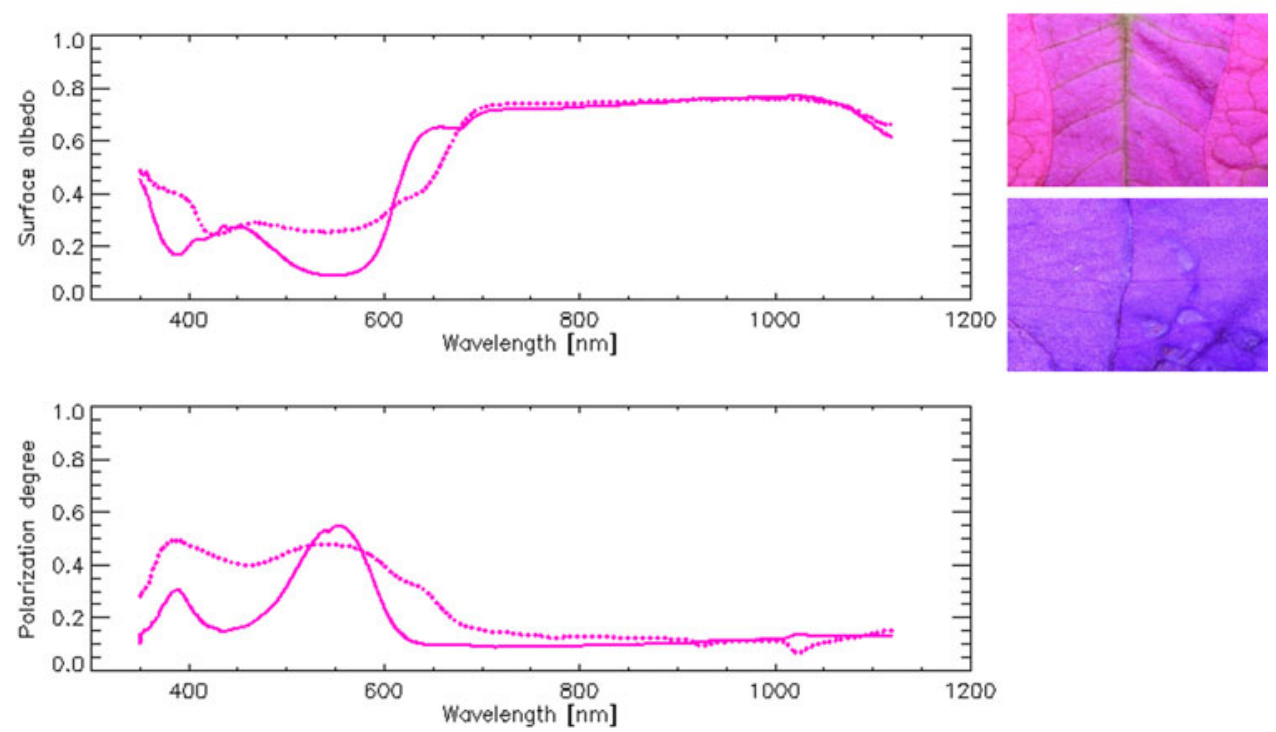

Fig. 5. Reflectance and polarized spectra of a purple leaf with green inclusions and of a purple flower petal (images of the samples top to bottom): L8-solid, P4-dotted (see Table 1). The presence of chl is seen as a small absorption feature near $670 \mathrm{~nm}$, while other biopigments absorb yellow and green and reflect red and blue to make the purple colour.

(when disregarding variations of specular reflection due to different leaf glossiness). This favourable occurrence allowed us to extract average polarized spectra corresponding to a given type of pigments. They were used to model planetary signals in Section 'Polarized spectra of habitable earth-like planets'.

Another remarkable feature is that the maximum polarization is associated with the absorption features, i.e. photons that escaped the absorption (reflected) are highly polarized. We gather that this is a pure radiative transfer effect within the upper, semi-transparent layer of the sample. Indeed, photosynthesis is a highly quantum efficient process. Normally, more than $90 \%$ of photons can be absorbed at a given wavelength. This also includes multiply-scattered photons with reduced polarization, which eventually get absorbed. However, those few per cent of photons, which escaped the absorption, will be scattered within the top, optically thin layer most probably only 1-2 times, and this light will be highly polarized. The photons escaping the leaf from the back side (for semi-transparent samples) were not measured in our experiment. In the landscape situation those photons will contribute to diffuse, unpolarized background light which may be 

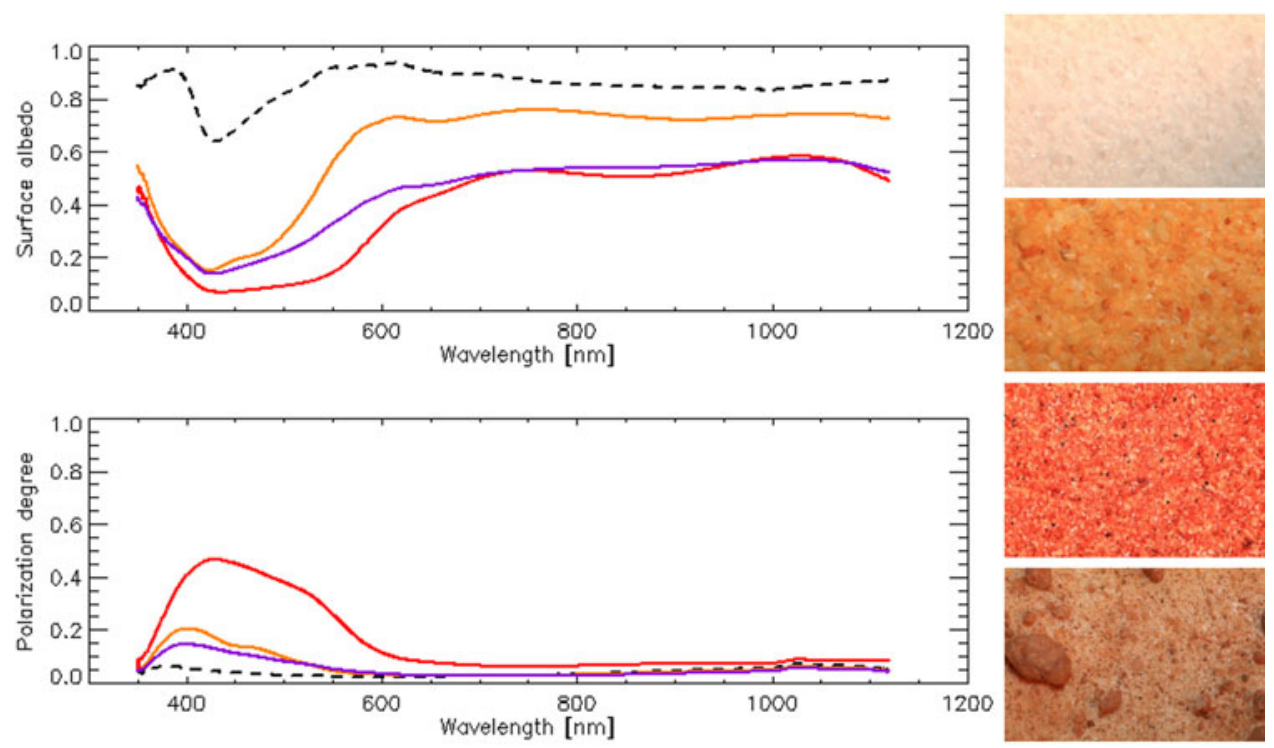

Fig. 6. Reflectance and polarized spectra of various sands (images of the samples top to bottom): S1-black dashed, S2-yellow, S3-red, S4-purple (see Table 1). Note that absorption features in the reflectance correspond to the colour of the sand, while polarization is significantly less prominent.

eventually absorbed by shadowed leaves/plants. An analysis of the angular dependence of the polarization of the reflected light can provide a single-particle albedo and the size of the scattering particles within the sample. To carry out such an analysis is the goal of our forthcoming paper. The high linear polarization associated with the absorption features of biopigments is a powerful biosignature for remote (or non-invasive) detection of life, which we investigate in this paper.

We also note that while the overall shape of the spectral features strongly depends on the presence of a particular pigment, the maximum polarization also depends on the glossiness of the sample. The elevated polarization is a specular reflection effect and is seen white in sample images, but the spectral signature is well preserved, especially for rough surface leaves. Smooth, flat leaves can be seen almost completely white in specular reflection. However, natural leaf surface is not flat, so in landscapes only some leaf surface parts will be seen in specular reflection. In fact, the signal measured from landscapes contains contributions from different reflection angles depending on the leaf orientation toward the stellar light. Therefore, realistic landscape signal modelling requires lab BRDF-PS measurements and averaging over angles (see Section 'Polarized spectra of habitable earth-like planets').

We have found that (1) plants with the same pigments demonstrate similar spectral dependence of reflectance and polarization, and (2) different pigments produce very different polarized spectral signatures associated with their selective absorption ability especially if several types of biopigments are present in the same sample. Only pure red hibiscus flower petal resembles in shape the red sand signature but it still differs by a larger polarization degree. Thus, in most cases we can distinguish biopigments and confirm their biological origin through polarimetric measurements.

\section{Inorganic samples}

We also measured polarized spectra of non-biological samples, such as sands and rocks from various parts of the worlds (Sahara, Australia, North America, Hawaii, see Table 1). By comparing polarized spectra of biological samples with the spectra of sands and rocks we looked for criteria to distinguish biological and non-biological pigments. Their reflectance and polarization spectra are presented in Figs. 6 and 7.

The characteristic absorption signatures of the plant biopigments are very different in shape from inorganic pigments found in sands and rocks, especially when reflectance is combined with polarimetric signals and several biopigments are present. This combination makes it robust to distinguish between biological and non-biological pigments. We note however, that radiative scattering effects on sand and rock grains are also present, and they are dependent on the grain size and rock composition. Again, we can extract this dependence using angular dependence of BRDF-PS.

\section{Polarized spectra of habitable earth-like planets}

We used our measurements (both reflectance and polarization spectra) presented in Section 'Inorganic samples' for modelling polarized and flux spectra of planets with Earth-like atmospheres. In order to investigate the sensitivity of biopigment detection, we assumed different surface coverage for photosynthetic organisms, ocean, deserts (sand and rock) and clouds. We followed the approach of Stam (2008) and used atmosphere, cloud and ocean spectra from that work. Present and new future observations of Earth-like planets around distant stars will not be able to resolve the planet surface and image its structures directly. However, uneven 

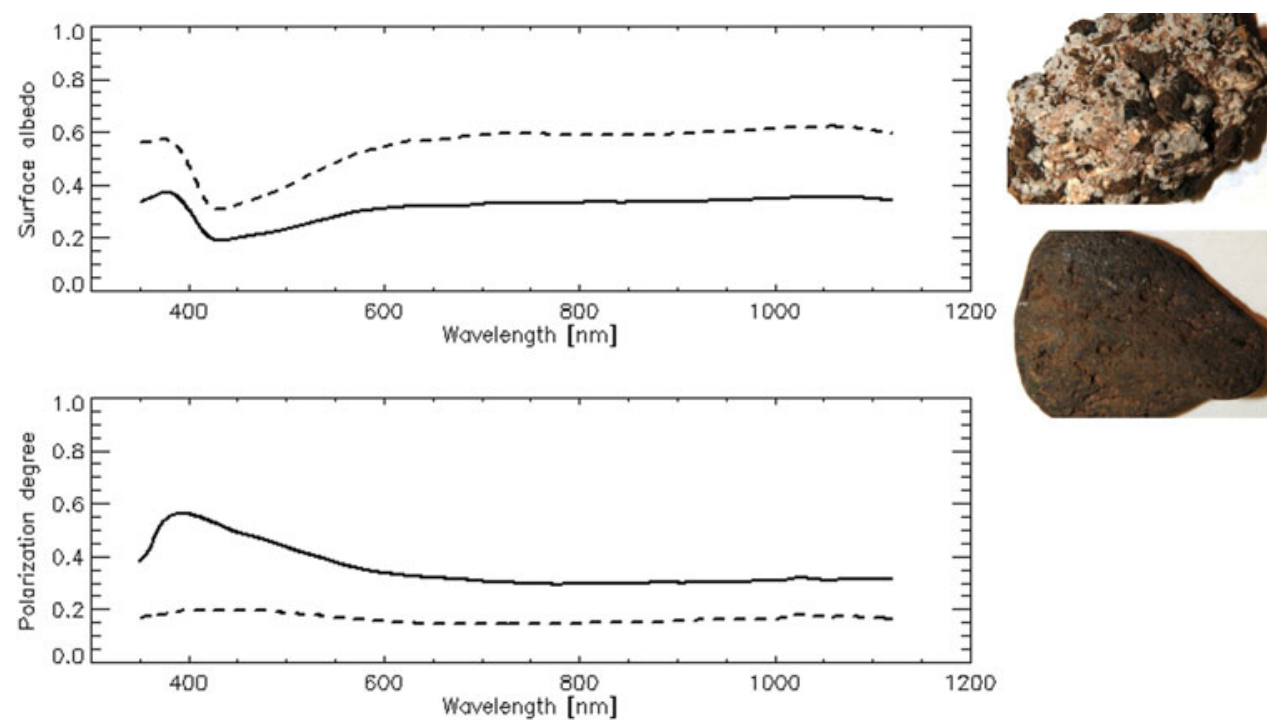

Fig. 7. Reflectance and polarized spectra of rocks (images of the samples top to bottom): R1-dashed, R2-solid (see Table 1). Again, polarization is significantly less prominent and weakly wavelength dependent as compared with biological samples.

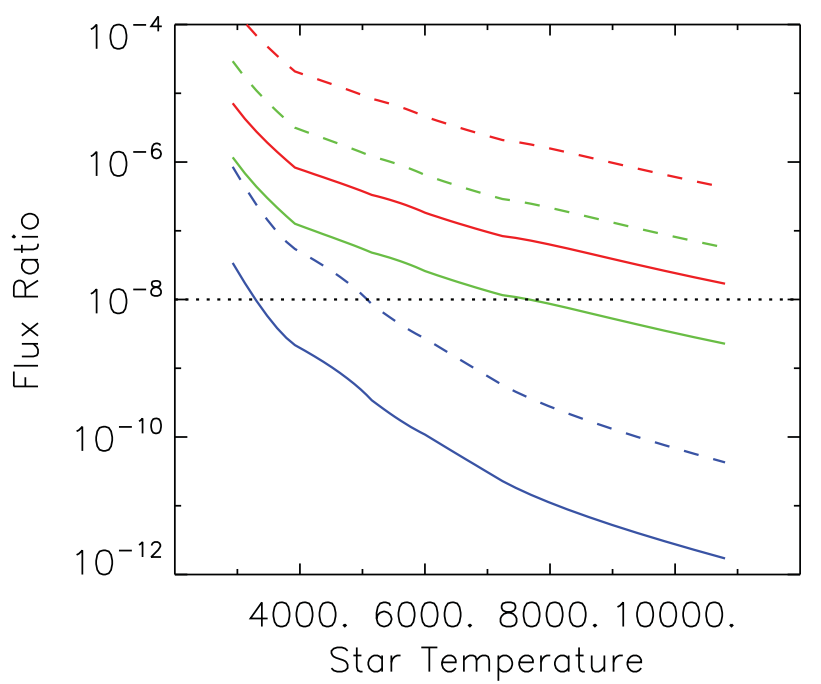

Fig. 8. Flux contrast for a planet in the habitable zone versus star temperature in scattered stellar light (blue), in planet emission at the wavelength of $5 \mu \mathrm{m}$ (green) and in emission at $10 \mu \mathrm{m}$ (red). Solid lines show contrast of Earth-radius planets and dashed lines correspond to five Earth-radius planets. The Earth-like optical geometrical albedo 0.3 was assumed. From Kuhn \& Berdyugina (2015).

distribution of land masses and their various surface properties as on Earth seen from space will produce rotational modulation of the reflected light which can be detected and used to constrain the overall surface coverage of various components. The contrast we have to achieve for detecting light from Earth-like planets in habitable zones depends on the spectral class of the star and wavelength (Fig. 8). It is feasible that the contrast down to $10^{-8}$ can be achieved with the current technology. However, to collect the necessary amount of photons in order to achieve such a high contrast for small planets requires extremely large telescopes. It appears that $25-40 \mathrm{~m}$ telescopes will be able to see only a few such planets. Large telescopes, such as the $75 \mathrm{~m}$ Colossus telescope are needed to investigate hundreds of Earth-like planets in stellar habitable zones (Kuhn \& Berdyugina, 2015), but even such large telescopes will be able to detect their light with a sufficient SNR at very low spectral resolution or in broad bands. The fact that absorption and polarization features of bioigments are extremely broad allows for filters designed to provide enough detail on their possible photosynthetic origin.

Our modelled spectra are shown in Figs. 9-12. To model these spectra we used only four biopigment samples (L5green, L6d-red, P1-yellow, L8-purple) and all but one (except white sand) inorganic samples. The curve colours in Figs. 9-12 correspond to the colours of the samples. We present here models for the following four illustrative cases:

(1) $100 \%$ coverage by organisms carrying different biopigments (Fig. 9, curve colours correspond to organism colours presented in Figs. 2-5): this example demonstrates the overall high sensitivity of polarimetric measurements to each assembly of biopigments, in contrast to flux measurements. In the visible part of the spectrum, the flux is reduced by $20-30 \%$ due to biopigment absorption, while their polarization reaches $30-80 \%$. The red part of the spectrum $(>700 \mathrm{~nm})$ indicates the presence of water and oxygen in the terrestrial atmosphere. The expected maximum polarization degree for the organisms containing Chl seen through the Earth-like atmosphere agrees well with the POLDER satellite measurements of the cloud-free forest canopy: $60 \%$ at $443 \mathrm{~nm}, 28 \%$ at $670 \mathrm{~nm}$ and $7 \%$ at $865 \mathrm{~nm}$ (Wolstencroft \& Breon 2005; Wolstencroft et al. 2007). This comparison also illustrates that polarization cancelation due to different angles of leaf surfaces with respect to the light source and viewing angles is not a major problem, but this effect should be approximated for more precise modelling. Because of Rayleigh scattering in the 

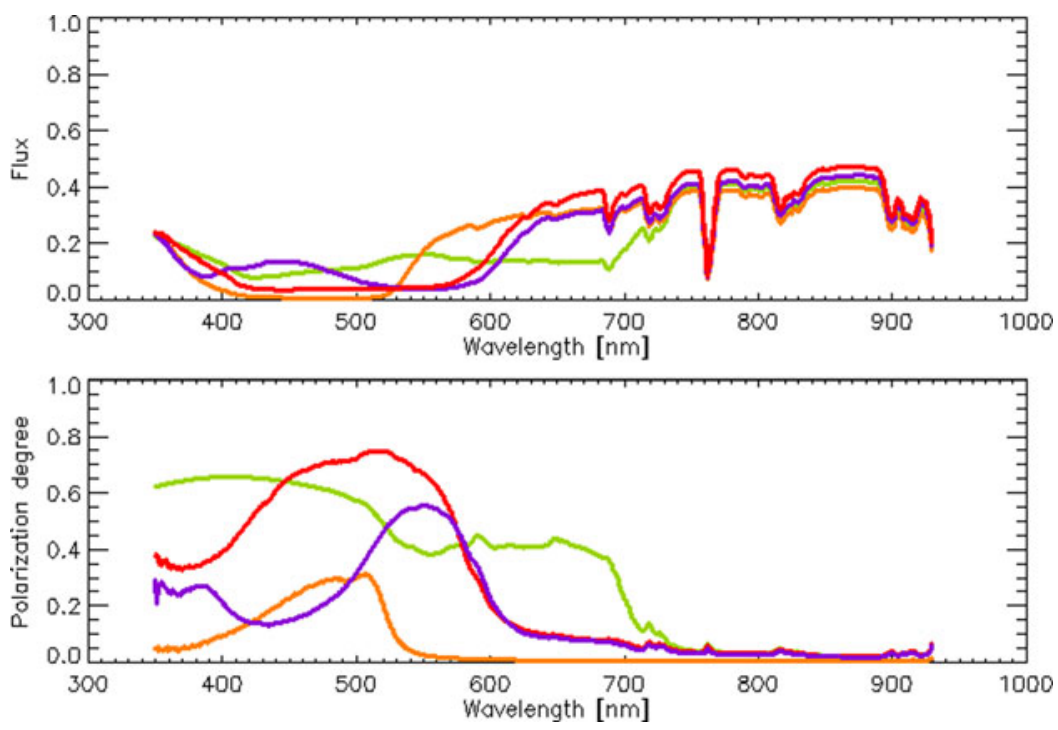

Fig. 9. Modelled reflectance spectra (top) and linear polarization degree spectra (bottom) for planets with the Earth-like atmosphere and $100 \%$ of surface coverage by pigmented organisms (visible hemisphere only). Colours of curves correspond to planet models with organisms carrying corresponding assemblies of biopigments shown in Figs. 2-5 (L5-green, L6d-red, P1-yellow, L8-purple). Notice the high linear polarization degree clearly distinguishing the presence of various biopigments in contrast to the flux spectra.
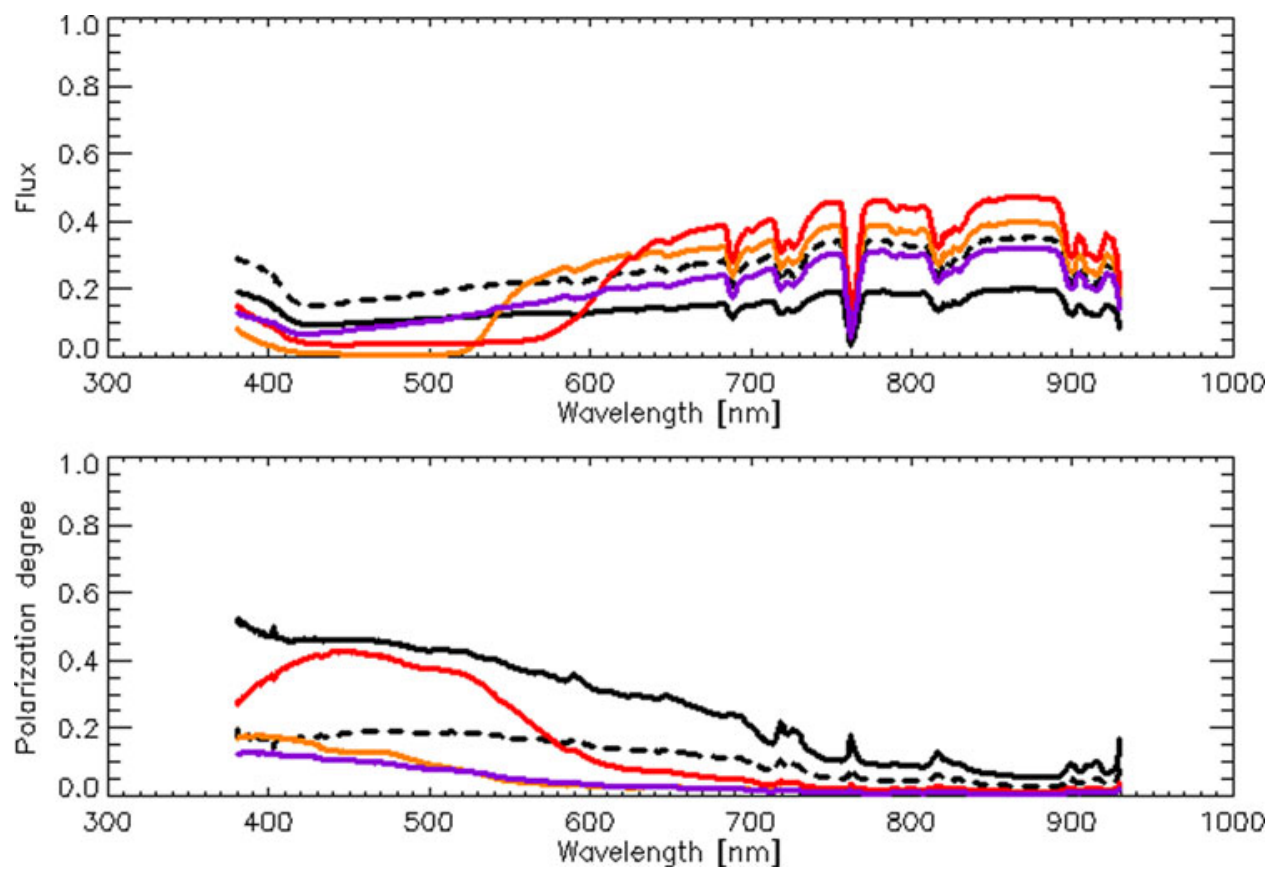

Fig. 10. The same as in Fig 9, but for a planet with $100 \%$ of desert coverage in the visible hemisphere. Colours of curves correspond to planet models with corresponding sands and rocks shown in Figs. 6 and 7. Note the relatively high polarization in the blue can help distinguishing the presence of various desert composition in contrast to the flux spectra. However, the polarization signal is different from that of biopigments of the same colour.

Earth atmosphere the reflected light gains a contribution in the blue part. This is exactly what is seen from space.

(2) $100 \%$ coverage by desert composed of different sands and rocks (Fig. 10): this example demonstrates quite good sensitivity of polarimetric measurements to the desert composition of landmasses in contrast to flux measurements, which do not show significant difference between the spectra except for yellow and red sands. In the visible part of the spectrum, the flux is reduced by $10-30 \%$ due to mineral absorption, and their polarization reaches 10 $50 \%$. The shape of the polarization curves is very different from those of biopigments (Fig. 9), except for the red sand, which can mimic pure red biopigments unless they are mixed with other biopigments (see Fig. 3). Again, our 

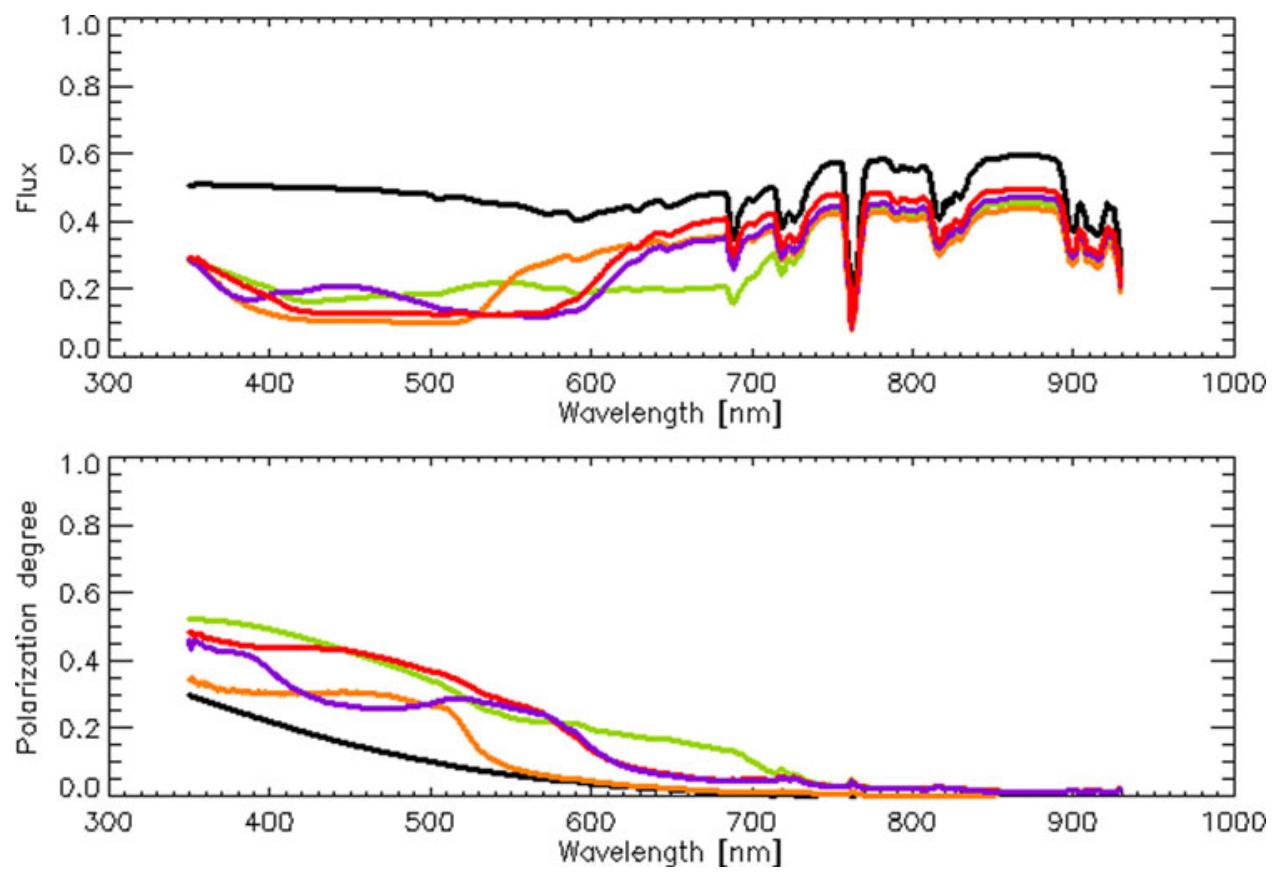

Fig. 11. The same as Fig. 9, but for a planet with $80 \%$ surface coverage by pigmented organisms and $20 \%$ cloud coverage (visible hemisphere only). Black curves represent a planet with 100\% cloud coverage (Stam 2008). In this particular case, the reflectance from the clouds is dominated by Rayleigh scattering and dominates the blue part of the spectrum. Obviously, the complete cloud coverage masks the property of the surface features.
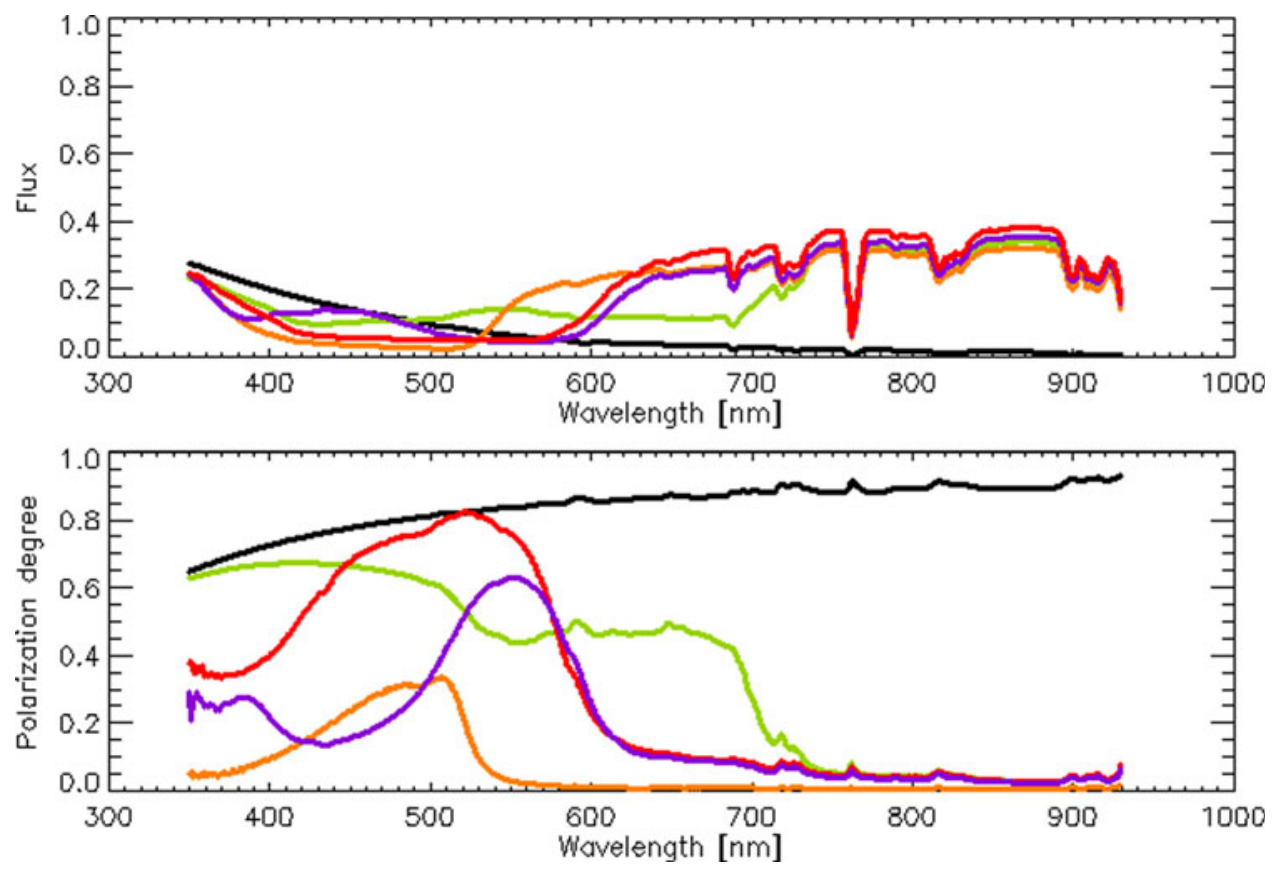

Fig. 12. The same as Fig. 9, but for a planet with $80 \%$ surface coverage by pigmented organisms and $20 \%$ ocean surface coverage (visible hemisphere only). Black curve represents a planet with an ocean only (Stam 2008). The reflectance from the ocean is dominated by reflection which is practically colour-independent.

expected maximum polarization degree for deserts seen through the Earth-like atmosphere agrees well with POLDER measurements of the cloud-free terrestrial deserts: $44 \%$ at $443 \mathrm{~nm}, 10 \%$ at $670 \mathrm{~nm}$ and $6 \%$ at $865 \mathrm{~nm}$ (Wolstencroft \& Breon 2005; Wolstencroft et al. 2007).
(3) $80 \%$ coverage by pigmented organisms and $20 \%$ by clouds (Fig. 11): this example demonstrates the diluting effect of clouds. A completely cloudy atmosphere will obviously disguise the presence of biopigments (and everything else) on the planet surface. However, small cloud coverage 
of around $20 \%$ will only marginally reduce polarization effect. Therefore, clouds are the most disturbing factor in detecting surface biosignatures, but weather variability and short life time of many cloud types should assist in successful detection if a planet is monitored long enough to reveal long-lived features on the surface.

(4) $80 \%$ coverage by pigmented organisms and $20 \%$ by clear ocean (Fig. 12): this example demonstrates the possible positive effect of the ocean on polarization measurements. The optical thickness of the ocean is basically infinite, so its surface is dark in most colours except for the blue, where it reflects the blue light scattered in the Earth's atmosphere. However, there is a bright glint at the subsolar location, which moves around the globe as the planet rotates. This glint is due to specular reflection and is highly polarized and practically white. Therefore, an ocean only, cloud-free planet with an Earth-like atmosphere will appear somewhat blue (due to Rayleigh scattering in the atmosphere) but highly polarized. If biopigments are present on the surface of landmasses, their polarization will be well visible (depending on the surface coverage percentage) in the same way as glossy leaves elevate the overall polarization but preserve spectral signatures of the biopigments. Therefore, this case, when the ocean is present and the atmosphere is optically thin enough to preserve surface polarization, seems the most favourable for remote polarimetric detection of exoplanets and biopigments.

We note that these examples are rather illustrative. The surface coverage percentage for a given signal certainly depends on modelling assumptions (e.g. atmosphere and cloud properties) as well as on the angles with respect to the illuminating star and the observer. In a subsequent paper we will use complete BRDF-PS sets to predict reflection curves for various planet orbital parameters. We note also that the goal of our modelling is to demonstrate the high sensitivity of photosynthetic biosignatures for remote detection of life when observed in linearly polarized light. As mentioned above, it is challenging to collect photons reflected off a distant Earth-like planet, and the achieved SNR will be most probably $\sim 10$ to $\sim 100$, leading to an error of about $10-1 \%$, respectively. Therefore, having the biosignature with linear polarization of $>50 \%$ will allow for its detection at a 5 - to 50 -fold error level. Detection of the circular polarization chiral biosignature of $0.05 \%$ requires SNR of at least 10000 (assuming 100\% coverage on the planet), which translates into the telescope size requirement of more than $8000 \mathrm{~m}$, and it is not feasible in the near future. Furthermore, using spectropolarimetry (or multiple broad-band filters) is necessary to avoid high constraints on the polarimetric precision. Our modelling demonstrates that simultaneous measurements at several wavelengths can be interpreted relative to each other in order to conclude on the presence of the biosignature. Understanding various instrumental effects is necessary for achieving polarization error of $1 \%$ or better (e.g. Harrington et al. 2014). This should be a goal for an astronomical instrument proposal to utilize our technique for remote detection of life.

\section{Prospects: from plants to microorganism}

Life arose in a very different environment compared with that of the modern Earth. A microfossil analysis of ancient rocks and sedimentological and geochemical information on the environmental conditions suggest that the Early Archaean microorganisms must have lived at high temperatures (thermophilic microorganisms), in water with salinities higher than the present day ocean (halotolerant and halophilic microorganisms), possibly under high UV exposures in extremely shallow water to subaerial environments ( $U V$ resistant microorganisms), or in dry environment (xerophilic microorganisms). Also, photosynthesis has appeared very early in the history of the Earth (Des Marais 2000), with complex land plants being relatively late arrivals. One can expect that photosynthetic microorganisms would also be among the first life forms on other planets. As photosynthetic pigments coming from plants are the same pigments that are present in microbial cells, we expect prominent polarized signatures associated with these pigments also in spectra of corresponding microorganisms. We also expect that variations of relative compositions of pigments can create unique features that will allow us to distinguish organisms through remote sensing or non-invasive analysis for both terrestrial and extraterrestrial life. Our successful investigation of biopigment polarization in plants encourages us to consider that the same biosignature is produced by bacteria and archaea, which use biopigments to both harvest stellar light as well as to protect themselves from the toxic radicals produced by UV radiation. We have already started employing our technique for extensive spectropolarimetric studies of microorganisms, and these results will be published as a continuation of the present study.

\section{Acknowledgements}

This work was supported by the SAW-2011-KIS-7 grant provided by the Leibniz Association, Germany, and the Advance Grant projects HotMol and ASTERISK provided by the European Research Council (grant agreements 291659 and 267864, respectively). Funding for the Stellar Astrophysics Centre is provided by The Danish National Research Foundation (Grant agreement no.: DNRF106). SB acknowledges support by the NASA Astrobiology Institute and Institute for Astronomy, University of Hawaii, USA. We thank anonymous referees for thorough reviews which helped to improve the manuscript.

\section{References}

Berdyugina, S.V. (2012). Polarimetric signatures of habitable planets. AbSciCon, \#1351741, http://abscicon2012.arc.nasa.gov/abstracts/abstractdetail/polarimetric-signatures-of-habitable-planets/

Berdyugina, S.V., Berdyugin, A.V., Fluri, D.M. \& Piirola, V. (2008). First detection of polarized scattered light from an exoplanetary atmosphere. Astrophys. J. Lett. 673, L83-L86.

Berdyugina, S.V., Berdyugin, A.V., Fluri, D.M. \& Piirola, V. (2011). Polarized reflected light from the exoplanet HD189733b: First 
multicolor observations and confirmation of detection. Astrophys. J. Lett. 728, L6-L10.

Beuzit, J.-L., Boccaletti, A., Feldt, M., Dohlen, K., Mouillet, D., Puget, P., Wildi, F., Abe, L., Antichi, J., Baruffolo, A., Baudoz, P., Carbillet, M., Charton, J., Claudi, R., Desidera, S., Downing, M., Fabron, C., Feautrier, P., Fedrigo, E., Fusco, T., Gach, J.-L., Giro, E., Gratton, R., Henning, T., Hubin, N., Joos, F., Kasper, M., Lagrange, A.-M., Langlois, M., Lenzen, R., Moutou, C., Pavlov, A., Petit, C., Pragt, J., Rabou, P., Rigal, F., Rochat, S., Roelfsema, R., Rousset, G., Saisse, M., Schmid, H.-M., Stadler, E., Thalmann, C., Turatto, M., Udry, S., Vakili, F., Vigan, A. \& Waters, R. (2010). Direct detection of giant extrasolar planets with SPHERE on the VLT, in Pathways towards habitable planets. ASP Conf. Ser. 430, 231-238.

Blankenship, E. \& Hartman, H. (1998). The origin and evolution of oxygenic photosynthesis. Trends Biochem. Sci. 23, 94-97.

Brakke, T. (1994). Specular and diffuse components of radiation scattered by leaves. Agricult. Forest Meteorol. 71, 283-295.

Breon, F.-M., Tanre, D., Lecomte, P. \& Herman, M. (1995). Polarized reflectance of bare soils and vegetation: measurements and models. IEEE Trans. Geos. Remote Sens. 33, 487-499.

Demory, B.-O., Seager, S., Madhusudhan, N., Kjeldsen, H., ChristensenDalsgaard, J., Gillon, M., Rowe, J.F., Welsh, W.F., Adams, E.R., Dupree, A., McCarthy, D., Kulesa, C., Borucki, W.J. \& Koch, D.G. (2011). The high albedo of the hot Jupiter Kepler-7 b. Astrophys. J. Lett 735, id. L12, 6pp.

Des Marais, D.J. (2000). When did photosynthesis emerge on Earth? Science 289(5485), 1703-1705.

Des Marais, D.J., Harwit, M.O., Jucks, K.W., Kasting, J.F., Lin, D.N.C., Lunine, J.I., Schneider, J., Seager, S., Traub, W.A. \& Woolf, N.J. (2002). Remote sensing of planetary properties and biosignatures on extrasolar terrestrial planets. Astrobiology 2(2), 153-181.

Evans, T.M., Aigrain, S., Barstow, J.K., Désert, J.-M., Gibson, N., Heng, K., Knutson, H.A. \& Lecavelier des Etangs, A. (2013). The deep blue color of HD 189733b: Albedo measurements with Hubble Space Telescope/Space Telescope imaging spectrograph at visible wavelengths. Astrophs. J. Lett 772, id. L16, 5pp.

Grant, L., Daughtry, C.S.T. \& Vanderbilt, V.C. (1993). Polarized and specular reflectance variation with leaf surface features. Physiol. Plant. 88, 1-9.

Harrington, D.M., Ftaclas, C., Chun, M., Berdyugina, S.V., Kuhn, J.R. \& Gisler, D. (2014). Cost effective polarimetric speckle suppression: the new Hokupa'a-85 curvature adaptive optics system and InnoPol EMCCD imaging polarimeter on the AEOS telescope. In Astronomical Telescopes and Instrumentation, Proc. SPIE, 9147, id. 91477C 19pp.

Hough, J.H., Lucas, P.W., Bailey, J.A., Tamura, M., Hirst, E., Harrison, D. \& Bartholomew-Biggs, M. (2006). PlanetPol: a very high sensitivity polarimeter. PASP 118, 1302-1318.

Jackson, J.D. (1998). Classical Electrodynamics. Wiley, New York, 3rd edn, 832pp.

Jacquemoud, S. \& Ustin, S.L. (2001). Leaf optical properties: a state of the art. In Proc. 8th Int. Symp. Physical Measurements \& Signatures in Remote Sensing, CNES, pp. 223-232.

Kemp, J.C., Henson, G.D., Steiner, C.T. \& Powell, E.R. (1987). The optical polarization of the sun measured at a sensitivity of parts in ten million. Nature 326, 270-273.

Kiang, N.Y., Siefert, J., Govindjee \& Blankenship, R.E. (2007a). Spectral signatures of photosynthesis I: review of Earth organisms. Astrobiology 7, 222-251.

Kiang, N.Y., Segura, A., Tinetti, G., Govindjee, Blankenship, R.E., Cohen, M., Siefert, J., Crisp, D. \& Measows, V.S. (2007b). Spectral signatures of photosynthesis II: coevolution with other stars and the atmosphere on extrasolar worlds. Astrobiology 7, 252-274.

Kuhn, J.R. \& Berdyugina, S.V. (2015). Global warming as a detectable thermodynamic marker of Earth-like extrasolar civilizations: a case for larger telescopes. Int. J. Astrobiology 14(3), 401-410.

Peltoniemi, J., Hakala, T., Suomalainen, J. \& Puttonen, E. (2009). Polarised bidirectional reflectance factor measurements from soil, stones, and snow. J. Quant. Spectrosc. Radiat. Transfer 110, 1940-1953.

Perrin, M.D., Duchene, G., Millar-Blanchaer, Fitzgerald, M.P., Graham, J.R., Wiktorowicz, S.J., Kalas, P.G., Macintosh, B., Bauman, B., Cardwell, A., Chilcote, J., De Rosa, R.J., Dillon, D., Doyon, R., Dunn, J., Erikson, D., Gavel, D., Goodsell, S., Hartung, M., Hibon, P., Ingraham, P., Kerley, D., Konapacky, Q., Larkin, J.E., Maire, J., Marchis, F., Marois, Ch., Mittal, T., Morzinski, K.M., Oppenheimer, B.R., Palmer, D.W., Patience, J., Poyneer, L., Pueyo, L., Rantakyrö, F.T., Sadakuni, N., Saddlemyer, L., Savransky, D., Soummer, R., Sivaramakrishnan, A., Song, I., Thomas, S., Wallace, J.K., Wang, J.J. \& Wolff, S.G. (2015). Polarimetry with the Gemini planet imager: methods, performance at first light, and the circumstellar ring around HR 4796A. Astrophys. J. 799, id. 182, 26 pp.

Piirola, V., Berdyugin, A. \& Berdyugina, S.V. (2014). DIPOL-2: A double image high precision polarimeter. In Astronomical Telescopes and Instrumentation, Proc. SPIE, 9147, id. 91478I 9pp.

Pospergelis, M.M. (1969). Spectroscopic measurements of the four Stokes parameters for light scattered by natural objects. Sov. Astron. 12, 973-977.

Raven, P.N., Jordan, D.L. \& Smith, C.E. (2002). Polarized directional reflectance from laurel and mullein leaves. Opt. Eng. 41, 1002-1012.

Scholes, G.D., Fleming, G.R., Olaya-Castro, A. \& van Grondelle, R. (2011). Lessons learned from nature about solar light harvesting. Nature Chem. 3, 763-774

Sparks, W.B., Hough, J.H., Kolokolova, L., Germer, T.A., Chen, F., DasSarma, S., DasSarma, P., Robb, F.T., Manset, N., Reid, I.N., Macchetto, F.D. \& Martin, W. (2009). Circular polarization in scattered light as a possible biomarker. J. Quant. Spectrosc. Radiat. Transfer 110, 1771-1779.

Sridhar, V.V., Singh, R.P., Mahtab, A. \& Ghosh, R. (2005). Crop polarisation behavior derived from multi-temporal ADEOS-POLDER data. J. Indian Soc. Remote Sens. 33, 339-344.

Stam, D.M. (2008). Spectropolarimetric signatures of Earth-like extrasolar planets. Astron. Astropys. 482, 989-1007.

Stenflo, J.O., Keller, C.U. \& Gandorfer, A. (2000). Anomalous polarization effects due to coherent scattering on the Sun. Astron. Astrophys. 355, 789-803.

Sterzik, M.F., Bagnulo, S. \& Palle, E. (2012). Biosignatures as revealed by spectropolarimetry of Earthshine. Nature 483, 64-66.

Suomalainen, J., Hakala, T., Puttonen, E. \& Peltoniemi, J. (2009). Polarised bidirectional reflectance factor measurements from vegetated land surfaces. J. Quant. Spectrosc. Radiat. Transfer 110, 1044-1056.

Wolstencroft, R.D. (1974). The circular polarization of light reflected from certain optically active surfaces. In Planets, Stars, and Nebulae: Studied with Photopolarimetry, IAU Coll. 23, ed. Gehrels, T., 495

Wolstencroft, R.D. \& Breon, F. (2005). Polarization of planet Earth and model Earth-like planets, in astronomical polarimetry: current status and future directions. ASP Conf. Ser. 343, 211-212.

Wolstencroft, R.D., Breon, F. \& Tranter, G. (2007). Polarization of light reflected from forest canopies on Earth with applications to Earth-like planets with realistic cloud cover. Bull. American Astron. Soc. 39, 106, \#09.06 